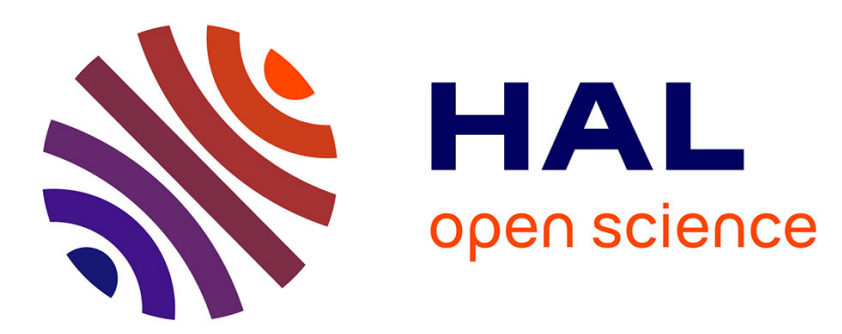

\title{
Multiresonant split ring resonator with meandered strips
} Jianping Hao, Amine-Rami Djouadi, François Rault, Xuyuan Tao, Eric Lheurette, Ludovic Burgnies

\section{To cite this version:}

Jianping Hao, Amine-Rami Djouadi, François Rault, Xuyuan Tao, Eric Lheurette, et al.. Multiresonant split ring resonator with meandered strips. physica status solidi (a), 2020, 217 (6), pp.1901017. 10.1002/pssa.201901017 . hal-03224089

\section{HAL Id: hal-03224089 \\ https://hal.science/hal-03224089}

Submitted on 11 May 2021

HAL is a multi-disciplinary open access archive for the deposit and dissemination of scientific research documents, whether they are published or not. The documents may come from teaching and research institutions in France or abroad, or from public or private research centers.
L'archive ouverte pluridisciplinaire HAL, est destinée au dépôt et à la diffusion de documents scientifiques de niveau recherche, publiés ou non, émanant des établissements d'enseignement et de recherche français ou étrangers, des laboratoires publics ou privés. 
This is the accepted version of the following article: J. Hao, A. Djouadi, F. Rault, X. Tao, É. Lheurette, and L. Burgnies, "Multiresonant Split Ring Resonator with Meandered Strips," physica status solidi (a), vol. 217, no. 6, p. 1901017, 2020, doi: https://doi.org/10.1002/pssa.201901017., which has been published in final form at https://onlinelibrary.wiley.com/doi/abs/10.1002/pssa.201901017

\section{Multi-Resonant Split Ring Resonator with Meandered Strips}

Jianping Hao, Amine Djouadi, François Rault, Xuyuan Tao, Éric Lheurette, and Ludovic Burgnies*

Dr. J. Hao, A. Djouadi, Prof. É. Lheurette

Univ. Lille, CNRS, Centrale Lille, Yncréa ISEN, Univ. Polytechnique Hauts-de-France, UMR 8520 - IEMN, F-59000 Lille, France.

Dr. F. Rault, Dr. X. Tao

ENSAIT, GEMTEX - Laboratoire de Génie et Matériaux Textiles

F-59000 Lille, France

Dr. L. Burgnies

Univ. Lille, CNRS, Centrale Lille, Yncréa ISEN, Univ. Polytechnique Hauts-de-France, UMR 8520 - IEMN, F-59000 Lille, France.

Also with Université du Littoral Côte d'Opale

F-62100 Calais, France

E-mail: ludovic.burgnies@univ-lille.fr

Keywords: metamaterials, multi-resonance, wearable technology, split-ring resonator, metamateials antenna

A multi-resonant split ring resonator (SRR) composed of two circular-shaped meandered strips is presented. Resonances are first analyzed by means of eigenmodes computation and field mapping. It is shown that a resonance can emerge from a standing wave taking place in each slot produced by a meandered strip. A slot operates as a two-wire transmission line section short-circuited at one termination and loaded by an open-circuit at the other end. Consecutively, a resonance occurs approximately when a quarter-wavelength matches the length of the slot. A resonance frequency shift is observed and it is related to a transverse magnetic coupling between a first anti-symmetric mode and a second symmetric mode. Finally, multiple resonances in an SRR are experimentally evidenced by 
spectroscopy of the input impedance of an SRR resonator antenna. Experimental results are favorably compared with simulation.

A split ring resonator (SRR) is commonly involved as an elementary cell for producing a magnetic response in metamaterials ${ }^{[1,2]}$. By means of a resonance, such SRR-based metamaterials allow to extend the permeability value of materials found in nature $\left(\mu_{r}>1\right)$ to values lower than 1 or even negative. A mu-negative material (MNG) and a negative-index material (NIM), by combining SRRs and another elementary cell producing a negative permittivity, contribute to the development of new microwave and optical components ${ }^{[3,4]}$. On the other hand, drawbacks of the resonant behavior of SRRs are a narrow bandwidth and a mono-band operation around the resonance frequency. If a strong resonance is a welcome feature for sensing ${ }^{[5]}$ can be a limitation for other applications such as electromagnetic (EM) wave absorbing and filtering. Different strategies for designing metamaterials with a broad bandwidth and operating at multiple frequency bands have been sought in literature. The first approach was to $\operatorname{stack}^{[6-9]}$ or to gather in the same plane ${ }^{[10-12]}$ resonators of different size and topology, each of them resonating either at a frequency close to others in order to improve the bandwidth ${ }^{[6,7,9,11,12]}$, or at separated frequency for a multi-band operation ${ }^{[8,10]}$. A second solution was to design a multi-resonant elementary cell achieved either by a combination of split rings ${ }^{[13,14]}$ or by nested resonators ${ }^{[15-19]}$. This second solution was often involved in producing multi-band operation but a bandwidth broadening for a metamaterial-inspired antenna was shown as well ${ }^{[17]}$. Fractal-inspired cells were also considered for both a multi-band operation ${ }^{[20]}$ and a bandwidth enhancement ${ }^{[21,22]}$ with application dedicated to ambient EM energy harvesting, nanoantenna and EM wave absorbing, respectively. Other characteristic requirements can be achieved by such fractal structures and a labyrinth metasurface showed a good angular robustness of the filtering behavior under oblique incidence ${ }^{[23]}$. Finally in the infrared wavelengths region, several resonances were experimentally evidenced in the reflection and transmission spectra measured for an array of SRRs ${ }^{[24]}$ and U-shaped resonators ${ }^{[25]}$. Observed resonances were interpreted as a standing wave plasmonic resonance occurring when an integer number of half-wavelengths was equal to the length of 
the resonator. Actually, standing wave resonances are not restricted to plasmon and similar resonances were shown in microwave for $\mathrm{SRRS}^{[26,27]}$. In this paper, we propose to extend this approach by introducing a split ring resonator made of a circular meandered strip aimed at producing multiresonances. Compared with the previously mentioned works, resonances are produced in the slots instead of on the metallic pattern. The geometry is presented first and resonances are analyzed by means of eigenmodes computation and field mapping. Then, resonances are experimentally highlighted by impedance spectroscopy of an SRR resonator antenna produced by embroidery.

An SRR with two circular-shaped meandered strips is illustrated in Figure 1a. Each of the split rings is formed by a meandered $0.2 \mathrm{~mm}$-thick metallic strip defined by a width $w_{l}=0.2 \mathrm{~mm}$, a gap $g_{l}=0.15 \mathrm{~mm}$ and a slot $s=4 \mathrm{~mm}$. The two rings are separated by a gap $g_{2}$ depending on the total width of a ring $w_{2}=0.9 \mathrm{~mm}$ and on the mean diameter $d_{\text {in }}$ and $d_{\text {out }}$ of the inner and outer ring, respectively.

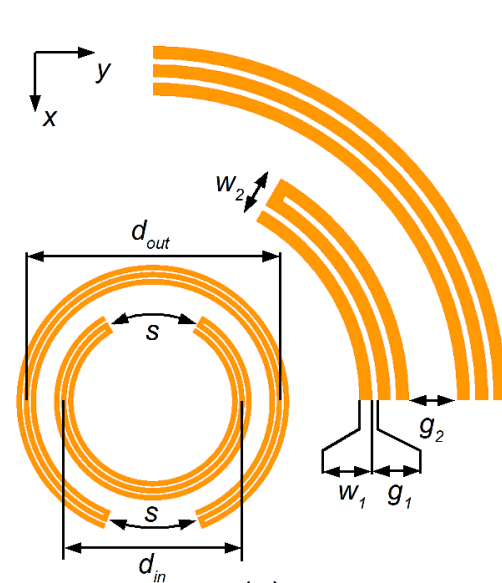

(a)

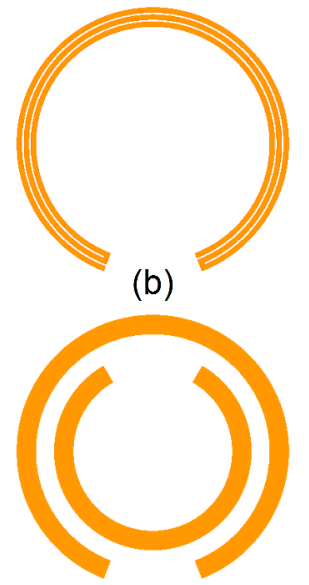

(c)

Figure 1. Geometry of a split ring resonator composed of two meandered strips (a), a single meandered strip (b) and two single strips (c).

Eigenmodes were computed by means of the commercial software HFSS by Ansys by considering a square lattice of SRRs of period $30 \mathrm{~mm}$ in the $x-y$ plane. As a boundary condition, a perfect magnetic conductor was put at $15 \mathrm{~mm}$ away from SRRs in the orthogonal $z$-direction in order to select relevant solutions with a magnetic field perpendicular to SRRs (transverse magnetic modes). The values of the resonance frequency and the quality factor ( $Q$-factor) calculated for different size and topology of an 
SRR are listed in Table 1. The SRR $a_{7.6}$ and $a_{8.0}$ corresponds to the resonator shown in Figure 1a with $d_{\text {out }}=10.8 \mathrm{~mm}$, and with $d_{\text {in }}=7.6 \mathrm{~mm}$ and $8.0 \mathrm{~mm}$, respectively. As illustrated in Figure $1 \mathrm{~b}$, the SRR $b_{7.6}, b_{8.0}$ and $b_{10.8}$ corresponds to a single ring with a meandered strip of diameter $d_{\text {out }}=7.6,8.0$, and $10.8 \mathrm{~mm}$, respectively. For such a resonator, the inner ring was removed before eigenmodes computation. At last, the SRR $c_{7.6}$ and $c_{8.0}$ corresponds to the double split rings shown in Figure $1 \mathrm{c}$ with $d_{\text {out }}=10.8 \mathrm{~mm}$, a width of each ring $w_{2}=0.9 \mathrm{~mm}$, and with $d_{i n}=7.6$ and $8.0 \mathrm{~mm}$, respectively.

Table 1. Resonance frequency in GHz and quality factor (in brackets) of SRRs and the SRR resonator antenna.

\begin{tabular}{cccccc}
\hline Structure & Mode 1 & Mode 2 & Mode 3 & Mode 4 & Mode 5 \\
\hline$a_{7.6}$ & $2.13(96.4)$ & $2.82(136)$ & $3.21(121)$ & $3.67(432)$ & $4.53(211)$ \\
$a_{8.0}$ & $2.13(96.2)$ & $2.81(136)$ & $3.02(118)$ & $3.39(405)$ & $4.29(208)$ \\
$b_{7.6}$ & $3.17(101)$ & $4.20(143)$ & & & \\
$b_{8.0}$ & $3.00(108)$ & $4.00(152)$ & & & \\
$b_{10.8}$ & $2.14(100)$ & $2.83(142)$ & & & \\
$c_{7.6}$ & $3.88(1313)$ & & & & \\
$c_{8.0}$ & $3.61(1259)$ & & & & \\
SRR resonator & 2.13 & 2.72 & 3.16 & 3.56 & 4.30 \\
antenna & & & & & \\
\hline
\end{tabular}

By comparing values in Table 1, we can see that the resonance frequency of the two first modes calculated for $a_{7.6}$ and $a_{8.0}$ are related to a resonance in the outer ring and the mode 3 and 5 to a resonance in the inner ring, whereas the mode 4 arises from a resonance between the two rings. The latter is similar to the resonance commonly observed in a double SRR with single strips (Figure 1c) which produces the highest value of the $Q$-factor (around 1300 in Table 1). The $Q$-factor of the SRR $a_{7.6}$ and $a_{8.0}$ is low (around 100-150) when the resonance is produced by the inner or the outer ring resonator (modes 1, 2, 3) whereas it is high $(Q>400)$ for the mode 4 involving the inter-ring resonance. This feature can be analyzed by considering a series $R L C$ circuit model equivalent to an SRR with a capacitance produced either between the two rings for the mode 4 or in the slot of a meandered strip for the other modes. As the gap $g_{1}$ is lower than $g_{2}$ and the slots are longer than the part of the two rings in facing, the equivalent 
capacitance involved in the mode 4 resonance is expected to be lower than the capacitance for the other modes. Consecutively, a higher $Q$-factor value results from $Q=1 /\left(R C \omega_{0}\right)$ where $\omega_{0}$ is the resonance angular frequency.

To gain further insight into the origin of the resonances, the current onto the metallic pattern is mapped in Figure 2 for five modes of the structure $a_{7.6 .}$. It is confirmed that the modes 1 and 2 are concerned with the outer ring and the modes 3 and 5 with the inner ring. For the mode 5, a current still remains on the outer ring as a signature of a coupling between the mode 4 and 5 . This coupling was also revealed in Table 1 with a resonance frequency and a $Q$-factor for the mode 4 and 5 deviating from values for the structures $c_{7.6}$ and $b_{7.6}$, respectively. On the contrary, for the modes 1,2 , and 3 only one ring is bright whereas the second is dark. For these modes, the SRR behaves as an isolated single ring resonator with the same values of the resonance frequency and $Q$-factor as shown in Table 1.

In Figure 2, we can see that the resonance mode 4 of $a_{7.6}$ is equivalent to the first mode of $c_{7.6}$, except a reverse direction of the current on the most inner strip. On the other hand, the mode 1 and 3 looks like a first standing wave plasmonic resonance with a current which is maximum at a half-length along the outer and inner ring, respectively ${ }^{[24-26]}$. Moreover, the mode 2 and 5 is similar to a second-order plasmonic resonance. As a general rule, such a resonance occurs when an integer number $m$ of halfwavelengths equals the length $L$ of the resonator $(L=m \lambda / 2)$. For a meandered ring of diameter $10.8 \mathrm{~mm}$, the first and second plasmonic resonance are evaluated to be 1.66 and $3.32 \mathrm{GHz}$ which are far away from the resonance frequency reported in Table 1 for $b_{10.8}$. Even if the half-wavelength matching condition can deviate due to the resonator geometry ${ }^{[28]}$ and to the skin effect ${ }^{[29]}$, a better explanation for resonances is required. 

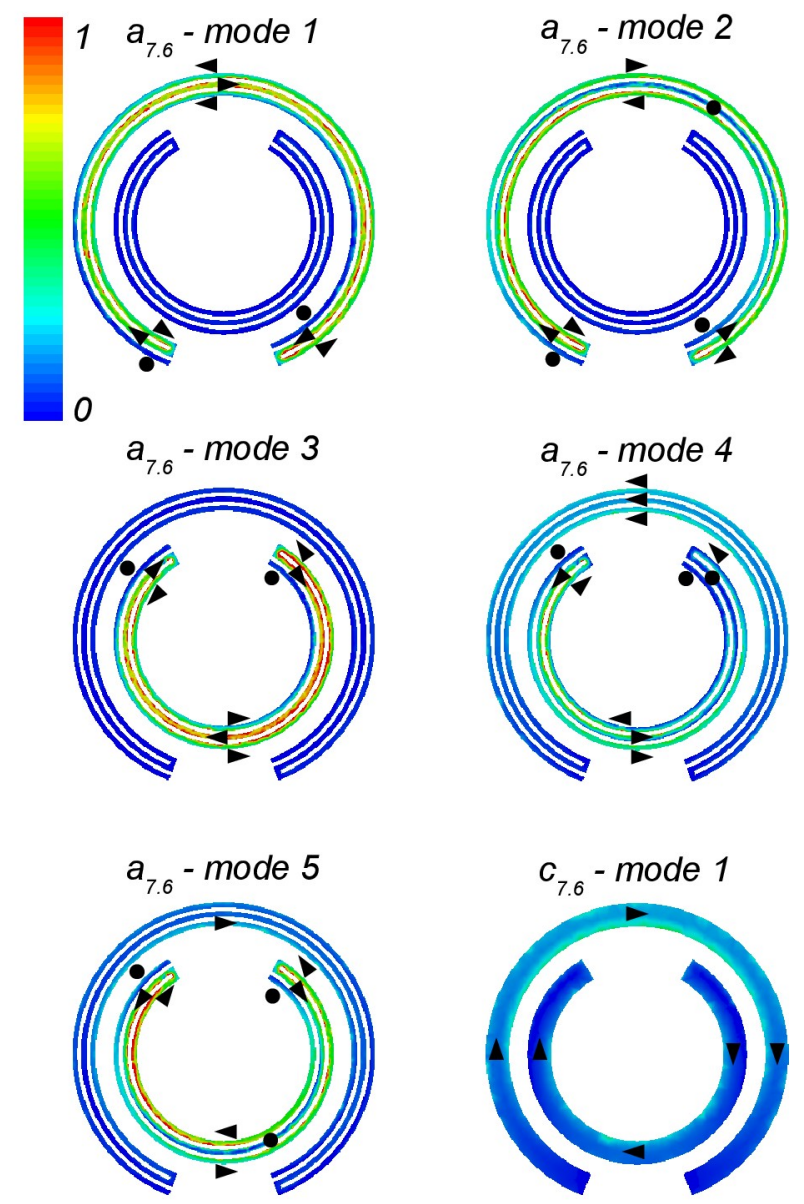

Figure 2. Normalized current map onto the metallic pattern of the SRR $a_{7.6}$ (modes 1 to 5 ) and $c_{7.6}$ (first mode). The direction of the current on each strip is indicated by an arrow and a zero current by a black dot.

In Figure 3, the electric-field ( $E$-field) is mapped for different modes in the plane of the SRR $a_{7.6}(z=0$ at a half-thickness of metallic strips). First, the mode 4 appears clearly as a resonance between the two rings. The $E$-field is also concentrated in the shorter slot of the inner ring which reflects the coupling between the modes 4 and 5. For all the other modes, the $E$-field is concentrated in a slot of a bright ring whereas the second ring is black. Then, the $E$-field is higher in the longest slot for the first resonance of a ring (modes 1 and 3) and is higher in the shortest slot for the second resonance (modes 2 and 5). At a resonance, the $E$-field is not uniform along a slot. The highest value is achieved at the open end of a slot whereas the $E$-field vanishes at the closed end. Conversely, the magnetic field (not shown for convenience) is maximum at the closed end and is low at the open end. It is also worth mentioning that the current orientation shown in Figure 2 points out a perpendicular magnetic field in the two slots of a 
ring in opposite directions for the modes 1 and 3 and in the same direction for the modes 2 and 5 . In conclusion, each resonance arising in a slot of a ring corresponds to an anti-symmetric mode (modes 1 and 3) or a symmetric mode (modes 2 and 5).

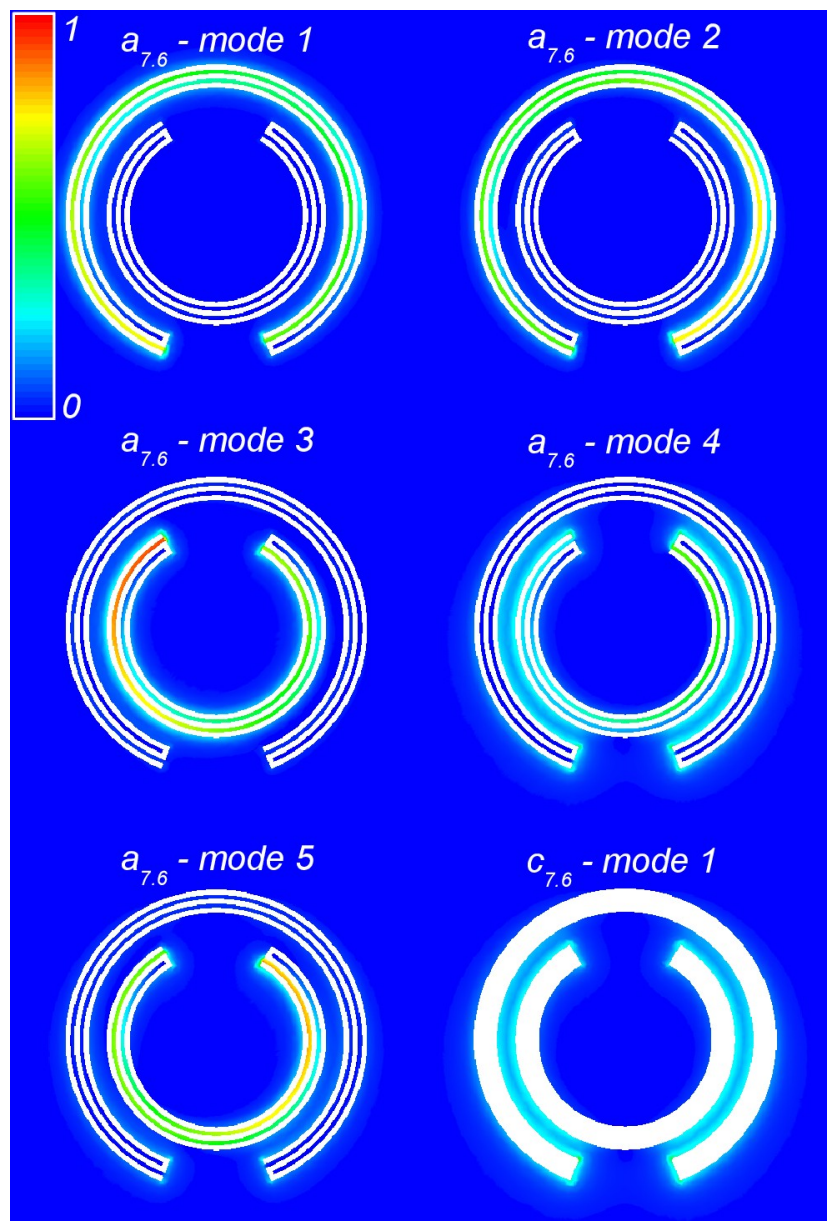

Figure 3. Normalized electric-field map for the SRR $a_{7.6}$ (modes 1 to 5) and $c_{7.6}$ (first mode).

By considering that a slot behaves as a two-wire transmission line section loaded by a short-circuit at one termination and by an open-circuit at the other end, the transmission line theory and the previous analysis of fields show that each resonance occurs approximately when a quarter-wavelength of the standing wave along a slot matches the length of the slot. This approach is similar to analytical solutions developed for explaining resonances in a singly split double ring $^{22}$. As the length of the two slots of a ring slightly differs, two resonances appear at close frequency. For $d_{\text {out }}=10.8 \mathrm{~mm}$ the quarterwavelength matching condition gives a resonance for the longest and the shortest slot at 2.44 and 
2.61 GHz, respectively. A shift of these resonance frequencies compared with values in Table 1 (2.14 and $2.83 \mathrm{GHz}$ for $b_{10.8}$ ) can result from both an imperfect open load and a coupling between modes. For the former, the open end of the slot is equivalent to a capacitive load and consecutively the resonance frequency decreases. Such a frequency shift has been estimated by complementary simulation of a single slot to be around $1.5 \%$. For the latter, it is well known that a coupling of resonators can produce a frequency shift. Particularly, electromagnetic interaction with two coupled SRRs was analyzed by means of electric and magnetic dipoles ${ }^{[30]}$. Depending on both the alignment of dipoles (symmetric or antisymmetric) and the dipole orientations (transverse or longitudinal) resonance modes can be differently split $^{[30,31]}$. The two resonances arising in the slots of a ring behave similarly to the transverse coupling of two magnetic dipoles with a first anti-symmetric mode and a second symmetric mode. Therefore, we can assume that the magnitude splitting of these resonances is mainly increased due to a transverse magnetic coupling between modes.

To experimentally assess multiple resonances in an SRR, a split ring resonator antenna ${ }^{[32]}$ was produced by embroidery. This technology is commonly considered for a textile production of wearable metamaterial antennas involving ring and split ring resonator ${ }^{[33-35]}$. It was conveniently selected in the presented work for energy harvesting application by smart textiles which is not specifically concerned with the present article. An S-shaped antenna with two SRRs is sketched in Figure 4a. It consists of an electric dipole made of a copper wire of diameter $0.5 \mathrm{~mm}$ and of two SRRs with previously regarded sizes corresponding to the mean values measured on the fabricated antenna shown in Figure $4 \mathrm{~b}$ and Figure 4c. The electric dipole was fixed onto a cotton fabric by embroidering polyester yarns. The thickness of the cotton fabric was measured to be $0.28 \mathrm{~mm}$ and the permittivity of various cotton fabrics measured $^{[36]}$ at $2.45 \mathrm{GHz}$ is $\varepsilon_{r}=1.5$. SRRs were fabricated by using a conductive yarn DataTrans from TIBTECH Innovations ${ }^{[37]}$ Company which was first triple passed in order to produce a meandered wire for each ring. Then, the meandered wire was fixed by embroidery with polyester yarns. The main advantage of this conductive yarn is a high conductivity compared to other conductive yarns used in 
embroidery ${ }^{[35]}$. A high conductivity is required to decrease the losses of the resonator. On the other hand, the yarn DataTrans is more rigid and a specific embroidery was developed to fix it on the cotton fabric. The reflection coefficient $\left(S_{11}\right)$ was measured by means of a Vector Network Analyzer after a preliminary calibration in the plane of the UFL connector and the input impedance of the antenna was calculated from $S_{11}$.

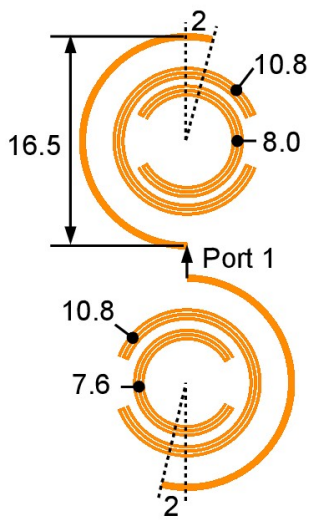

(a)

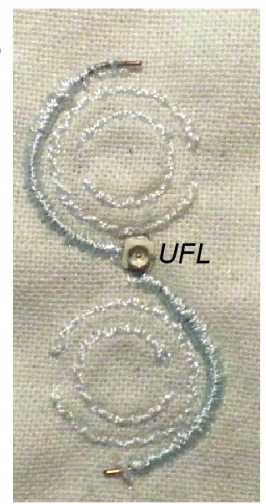

(b)

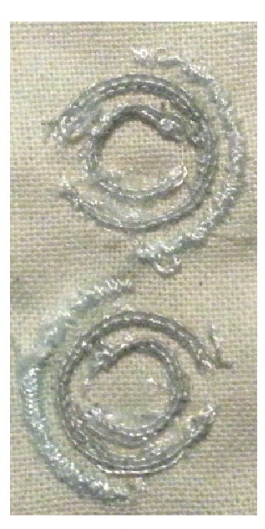

(c)

Figure 4. Illustration of the SRR resonator antenna: (a) simulated antenna (sizes in mm), (b) front side and (c) back side of the fabricated antenna. The antenna consists of an S-shaped dipole of diameter $16.5 \mathrm{~mm}$ and the SRRs $a_{7.6}$ and $a_{8.0}$. Each conductor of the dipole is $2 \mathrm{~mm}$ longer than a half-ring.

The measured reflection coefficient plotted in Figure 5 shows 7 dips revealing multiple resonances of the antenna. The impedance of the antenna is well matched with $50 \Omega$ at 2.5, 2.66, 3.38 and $3.63 \mathrm{GHz}$ with $S_{11}<-10 \mathrm{~dB}$ whereas $S_{11}>-5.4 \mathrm{~dB}$ for other three dips at $3.04,3.94$, and $4.42 \mathrm{GHz}$. We can note that the two first dips with close frequency lead to a broad bandwidth around $2.5 \mathrm{GHz}$. Moreover, a multiband operation of the antenna can be expected with two complementary dips around $3.5 \mathrm{GHz}$. 


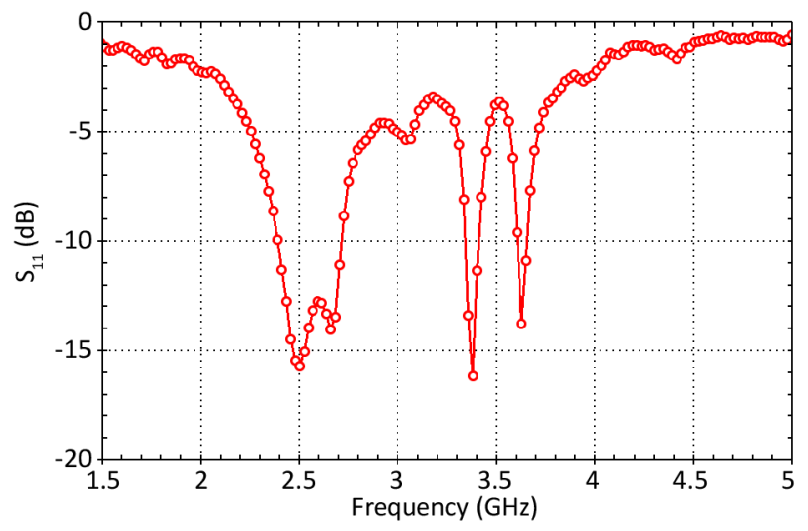

Figure 5. Measured reflection coefficient of the antenna. (Note circle marks have been added for all the experimental data)

To better analyze resonances, the real part of the measured input impedance is plotted in Figure 6. Between 2 and $4.5 \mathrm{GHz}$, we can see 6 resonances evidenced by a maximum of the impedance. These resonances are favorably compared in Figure 6 with the simulated impedance. However, simulation shows clearly 7 resonances and an eighth resonance can be revealed by a slight ripple of the impedance at around 3.2 GHz. All the resonance frequency values of the simulated antenna are reported in Table 1. Each of them can be unambiguously related to an eigenmode previously calculated for either SRR $a_{7.6}$ or $a_{8.0}$. Also, we can note that the most prominent resonances observed in Figure 6 stem from the modes 4 and 5 of each SRR which have the highest $Q$-factor values. However, the impedance spectrum was achieved by means of a dipole antenna and consecutively the amplitude of the resonances depends on the coupling between the dipole and the SRRs as well. By further cutting the dipole, its own resonance frequency increases and the coupling strength with SRRs changes. For the results plotted in Figure 5 and Figure 6, the length of the S-shaped dipole was profitably cut during measurements $2 \mathrm{~mm}$ longer than a half-ring allowing to bring out all the resonances in SRRs. The antenna performance was measured at the relevant frequency. The gain was $\mathrm{G}=2.7,0.45,-3$, and $-4.6 \mathrm{dBi}$ at $2.5,2.66,3.38$ and $3.63 \mathrm{GHz}$, respectively. A better gain than in the reference ${ }^{[35]}$ was achieved mainly thanks to a more conductive yarn used for the SRRs, but it is not possible to related this higher value to the meandered strip. 


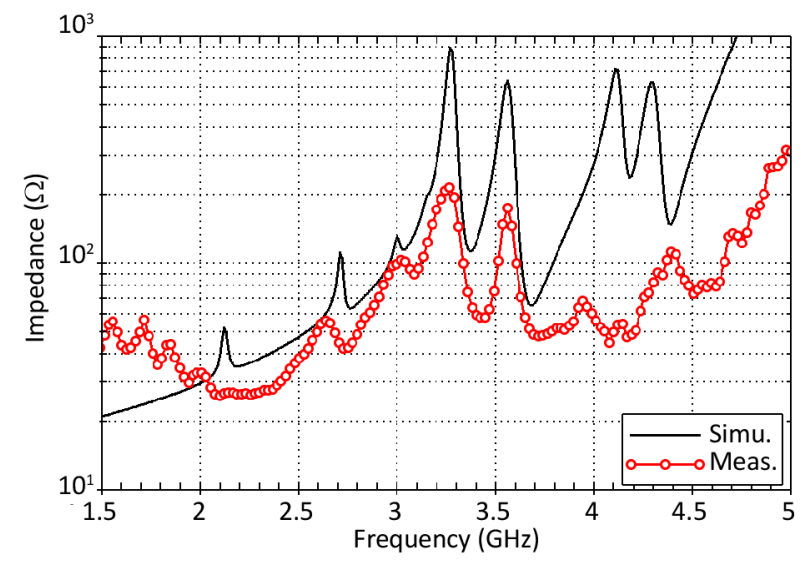

Figure 6. Real part of the input impedance of the SRR resonator antenna simulated (solid line with no mark) and measured (line with open circles). (Note circle marks have been added for all the experimental data)

In summary, multiple resonances of a split ring resonator made of two meandered strips have been analyzed by means of eigenmodes computation and field mapping. It has been shown that a slot in a meandered strip behaves as a two-wire transmission line section and a resonance occurs approximately when a quarter-wavelength of the standing wave along a slot matches the length of the slot. By combining two rings with two slots, five resonances result. Multiple resonances have been experimentally evidenced by spectroscopy of the input impedance of an SRR resonator antenna. Depending both on the quality factor of the resonance and the coupling strength between the dipole antenna and the SRRs, the impedance shows more or less prominent resonances. Such multiple resonances in an SRR produced by meandered strips can contribute to design metamaterials operating at multiple frequencies, opening new prospects for applications where a multiband operation is required.

\section{Acknowledgements}

This work was financially supported by the Fonds Européen de Développement Régional/ Met steun van het Europees Fonds voor Regionale Ontwikkeling in the framework of the European Interreg V FranceWallonie-Vlaanderen project named Luminoptex. 
Received: ((will be filled in by the editorial staff))

Revised: ((will be filled in by the editorial staff))

Published online: ((will be filled in by the editorial staff))

\section{References}

[1] D. R. Smith, W. J. Padilla, D. C. Vier, S. C. Nemat-Nasser, S. Schultz, Phys. Rev. Lett. 2000, 84, 4184.

[2] J. B. Pendry, A. J. Holden, D. J. Robbins, W. J. Stewart, IEEE Trans. Microw. Theory Tech. 1999, 47, 2075.

[3] J. B. Pendry, D. Schurig, D. R. Smith, Science 2006, 312, 1780.

[4] I. Gallina, G. Castaldi, V. Galdi, A. Alù, N. Engheta, Phys. Rev. B 2010, 81, 125124.

[5] M. Beruete, I. Jáuregui-López, Advanced Optical Materials 2019, 0, 1900721.

[6] S. Ji, C. Jiang, J. Zhao, J. Wang, H. Dai, physica status solidi (b) 2019, 0, 1900069.

[7] S. Liu, H. Chen, T. J. Cui, Appl. Phys. Lett. 2015, 106, 151601.

[8] S. Liu, J. Zhuge, S. Ma, H. Chen, D. Bao, Q. He, L. Zhou, T. J. Cui, J. Appl. Phys. 2015, 118, 245304.

[9] Y.-W. Zhu, L. Li, Y. Qiu, W.-T. Piao, Y.-J. Zhang, Appl. Phys. A 2017, 123, 361.

[10] N. Born, I. Al-Naib, C. Jansen, T. Ozaki, R. Morandotti, M. Koch, Appl. Phys. Lett. 2014, 104, 101107.

[11] Q. M. Nguyen, A. I. Zaghloul, T. K. Anthony, S. J. Weiss, IEEE Antennas Wireless Propag. Lett. 2019, $18,1026$.

[12] N. Fernez, L. Burgnies, J. Hao, C. Mismer, G. Ducournau, D. Lippens, É. Lheurette, IEEE Trans. Microw. Theory Tech. 2018, 66, 1764.

[13] Q.-Y. Wen, H.-W. Zhang, Y.-S. Xie, Q.-H. Yang, Y.-L. Liu, Appl. Phys. Lett. 2009, 95, 241111.

[14] S. Qiao, Y. Zhang, S. Liang, L. Sun, H. Sun, G. Xu, Y. Zhao, Z. Yang, J. Appl. Phys. 2015, 118, 123106.

[15] S. Hussain, J. Min Woo, J.-H. Jang, Appl. Phys. Lett. 2012, 101, 091103.

[16] X. Shen, Y. Yang, Y. Zang, J. Gu, J. Han, W. Zhang, T. Jun Cui, Appl. Phys. Lett. 2012, 101, 154102.

[17] S. Bhattacharjee, S. Maity, S. R. B. Chaudhuri, M. Mitra, IET Microwaves, Antennas and Propagation 2018, 12, 1799.

[18] Q. Ye, Y. Liu, H. Lin, M. Li, H. Yang, Appl. Phys. A 2012, 107, 155.

[19] F. Bilotti, A. Toscano, L. Vegni, IEEE Trans. Antennas Propag. 2007, 55, 2258.

[20] X. Zhang, H. Liu, L. Li, Appl. Phys. Lett. 2017, 111, 071902.

[21] L. Rosa, K. Sun, S. Juodkazis, Phys. Status Solidi RRL 2011, 5, 175.

[22] N. Fernez, Y. Arbaoui, A. Maalouf, A. Chevalier, P. Agaciak, L. Burgnies, P. Queffelec, V. Laur, É. Lheurette, J. Appl. Phys. 2018, 123, 084902.

[23] I. Jáuregui-López, P. Rodríguez-Ulibarri, A. Urrutia, S. A. Kuznetsov, M. Beruete, physica status solidi (RRL) - Rapid Research Letters 2018, 12, 1800375.

[24] C.-Y. Chen, S.-C. Wu, T.-J. Yen, Appl. Phys. Lett. 2008, 93, 034110.

[25] F. J. Rodríguez-Fortuño, R. Ortuño, C. García-Meca, J. Martí, A. Martínez, J. Appl. Phys. 2012, 112, 103104.

[26] J. García-García, F. Martín, J. D. Baena, R. Marqués, L. Jelinek, J. Appl. Phys. 2005, 98, 033103.

[27] T. Shaw, D. Mitra, Appl. Phys. A 2018, 124, 348.

[28] B. N. Khlebtsov, N. G. Khlebtsov, J. Phys. Chem. C 2007, 111, 11516. 
[29] F. Neubrech, T. Kolb, R. Lovrincic, G. Fahsold, A. Pucci, J. Aizpurua, T. W. Cornelius, M. E. Toimil-Molares, R. Neumann, S. Karim, Appl. Phys. Lett. 2006, 89, 253104.

[30] S. S. Seetharaman, C. G. King, I. R. Hooper, W. L. Barnes, Phys. Rev. B 2017, 96, 085426.

[31] N. Liu, H. Giessen, Angewandte Chemie International Edition 2010, 49, 9838.

[32] K. B. Alici, E. Ozbay, J. Appl. Phys. 2007, 101, 083104.

[33] B. Moradi, M. Martinez, R. Fernández-García, I. Gil, physica status solidi (a) 2018, 215, 1800410.

[34] I. Gil, R. Seager, R. Fernández-García, physica status solidi (a) 2018, 215, 1800377.

[35] J. Hao, A. Leblanc, L. Burgnies, A. Djouadi, C. Cochrane, F. Rault, V. Koncar, É. Lheurette, Electronics Letters 2019, 55, 508.

[36] S. Sankaralingam, B. Gupta, IEEE Transactions on Instrumentation and Measurement 2010, 59, 3122.

[37] "TIBTECH: SMART and conductive textiles, yarns or fabrics," can be found under https://www.tibtech.com/, 2019. 